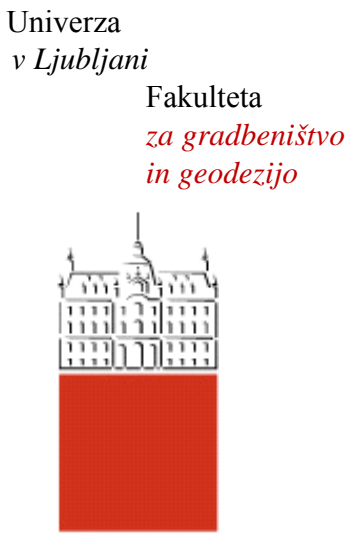

Jamova cesta 2

1000 Ljubljana, Slovenija

http://www3.fgg.uni-lj.si/

\section{DRUGG - Digitalni repozitorij UL FGG http://drugg.fgg.uni-lj.si/}

Ta članek je avtorjeva zadnja recenzirana različica, kot je bila sprejeta po opravljeni recenziji.

Prosimo, da se pri navajanju sklicujte na bibliografske podatke, kot je navedeno:
University
of Ljubljana

Faculty of

Civil and Geodetic

Engineering

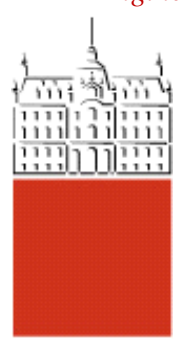

Jamova cesta 2

SI - 1000 Ljubljana, Slovenia

http://www3.fgg.uni-lj.si/en/

DRUGG - The Digital Repository http://drugg.fgg.uni-lj.si/

This version of the article is author's manuscript as accepted for publishing after the review process.

When citing, please refer to the publisher's bibliographic information as follows:

Kroflič, A., Saje, M., Planinc, I., Zupan, D. 2011. Buckling of asymmetrically delaminated three-dimensional composite beam: Analytical solution. Composites Part B-Engineering 42,7: 2047-2054 DOI: 10.1016/j.compositesb.2011.04.038. 


\title{
Buckling of asymmetrically delaminated three-dimensional composite beam: analytical solution
}

\author{
A. Kroflič ${ }^{\mathrm{a}}$, M. Saje ${ }^{\mathrm{a}}$, I. Planinc ${ }^{\mathrm{a}}$, D. Zupan ${ }^{\mathrm{a}, *}$ \\ ${ }^{a}$ University of Ljubljana, Faculty of Civil and Geodetic Engineering, Jamova 2, SI-1115 \\ Ljubljana, Slovenia
}

\begin{abstract}
The present paper presents a consistent model of a three dimensional delaminated composite column with a proper consideration of the extensional and bending stiffness coupling and transverse shear effect to determine the axial buckling load. The exact analytical solution of the buckling force is obtained using the linearized stability theory. Three dimensional model allows us to consider a rather general set of delaminations including those that are not necessarilly perpendicular to the symmetry axis of the cross-section or/and have non-symmetrical surfaces. The parametric studies are presented showing the effects of shear, the delamination position, the angle of rotation of the delamination and the ratio of elastic to shear moduli.
\end{abstract}

Keywords: B. Buckling, B. Delamination, C. Analytical modelling, Three-dimensional beam

\section{Introduction}

A proper design of composite structures demands a good understanding of their behaviour when subject to mechanical loads, and the mechanism of their collapse. One of the grounds for the collapse of laminated composite structures is their delamination, which may be caused by an air entrapment, a local lack

\footnotetext{
${ }^{*}$ Corresponding author

Email address: dejan.zupan@fgg.uni-lj.si (D. Zupan)
}

Preprint submitted to Elsevier

April 14, 2011 
of resin or other defects originating from a technological procedure, an impact or a high stress concentration.

Buckling is often a reason of collapse of the delaminated structural elements subject to compressive forces. That is why the behaviour of delaminated structures in compression has been investigated extensively during the last 30 years, using means fields of mathematical modelling including analytical solutions $([13],[17],[21])$, finite element analyses (FEA) ([10],[19]), experimentations and fracture mechanics-based researches $([6],[18],[22])$.

First reports on the behaviour of the delaminated structural elements appear in the seventies [1]. The initial insight into the buckling behaviour and the delamination growth was given by Chai et al. [3]. A similar, one dimensional model was proposed by Simitses et al. [19] to study the effect of the delamination length and position. Kordomateas and Schmueser [? ] developed a formulation which incorporated the effect of the transverse shear on the critical buckling force. A similar model was employed by Chen [4] to consider beams with two delaminations. Later on Moradi and Taheri [13] solved the same problem with an alternative numerical method called the quadratic differential method. Any of these procedures considers the effect of shear, only inconsistently by introducing correction factors and neglecting coupling of axial and transverse deformations. Kryžanovski et al. [11] presented buckling of asymmetrically single-delaminated, shear-deformable elastic columns based on the planar Reissner beam theory. Rodman et al. [17] extended the approach in [11] to columns with multiple delaminations. To the best knowledge of the authors, the analytical solutions for buckling of delaminated beams found in open literature have been limited only to planar problems. By contrast, the present paper will consider the delaminated beams in three dimensions. Such an approach will also allow us to consider asymmetrical cross-sections and a rather general set of delaminations including those that are not necessarilly perpendicular to any plane of the beam. The incorporation of the transverse shear effect and the coupling of the extensional and bending stiffnesses follows directly from the three-dimensional Reissner beam theory [15]. 
The linearized stability theory is employed to obtain the exact analytical solution of the buckling force without any simplifications assumed in the governing equations. The post-buckling analysis is, however, beyond the scope of the present paper. For the sake of simplicity the homogeneous linear elastic material is presumed in all layers, but the generalization of the formulation to composites made of several different materials is straightforward. The present approach is capable of considering a complex three-dimensional behaviour, where, e.g. the layer of the beam buckles locally in a direction different than the global deformation of the beam. It is pointed out that the shapes of cross-sections and delaminations are arbitrary.

\section{Problem definition}

We consider a straight three-dimensional beam with a constant cross-section, subject to a compressive axial force $F$ acting along the neutral axis of the beam (Figure 1), termed the column in the sequel. The column is delaminated by a single delamination plane A-B-C-D at an arbitrary position. The delamination divides the column into four elements: elements $\boldsymbol{a}$ and $\boldsymbol{d}$ with lengths $L_{1}$ and $L_{4}$, respectively, represent the perfect non-delaminated portions of the beam, while elements $\boldsymbol{b}$ and $\boldsymbol{c}$ (with equal length $L_{23}$ ) represent the two layers separated by the plane A-B-C-D. They represent the delaminated part of the column.

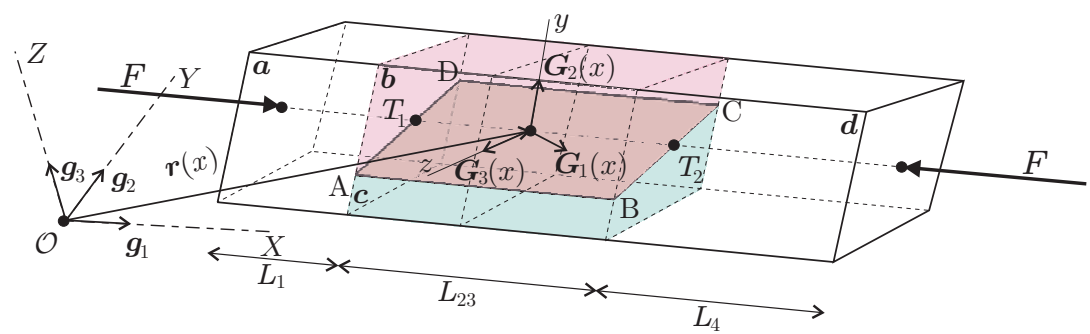

Figure 1: Geometry, loading and elements of a column. 


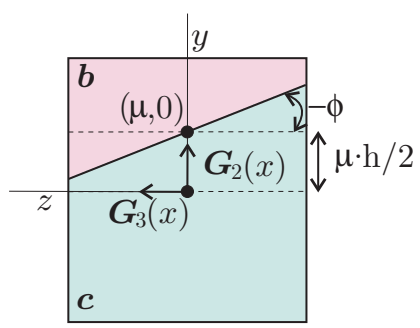

Figure 2: Definitions of $\mu$ and $\phi$.

The quantities, describing the column, will be expressed with respect to the arc-length parameter " $x$ " of the undeformed neutral axis. An arbitrary configuration of the column is described by the position vector $\boldsymbol{r}$ of the centroidal axis and the orthonormal base vectors $\left\{\boldsymbol{G}_{1}, \boldsymbol{G}_{2}, \boldsymbol{G}_{3}\right\}$ that span the planes of the cross-sections. Unit vectors $\boldsymbol{G}_{2}$ and $\boldsymbol{G}_{3}$ point along the principal axes of inertia of the cross-section, and $\boldsymbol{G}_{1}$ is the cross-sectional unit normal, $\boldsymbol{G}_{1}=$ $\boldsymbol{G}_{2} \times \boldsymbol{G}_{3}$. Due to shear deformations, $\boldsymbol{G}_{1}$ is not necessarilly tangential to the centroidal axis. The base vectors $\boldsymbol{G}_{i}$ define the local basis. It is also suitable to introduce the reference point $\mathcal{O}$ in the physical space, fixed global coordinate system $(X, Y, Z)$. The position of the delamination plane is uniquely defined by the parameters $\mu$ and $\phi$ as shown in Figure 2. An asymmetrical position of the delamination with respect to the local axis along vector $\boldsymbol{G}_{2}$ is defined by parameter $\mu \in(-1,1)$ see Figure 2 where, $h$ denotes the height of a cross section. For a vertically symmetrical delamination, parameter $\mu=0$. Increasing (decreasing) a value of $\mu$ reduces the thickness of one layer and increases the thickness of the other. It is also allowed for the delamination to rotate in a plane of the cross-section around the point $y=\mu, z=0$. The rotation is described by angle $\phi$.

\subsection{Governing equations of the three-dimensional beam}

Our starting point is the set of the equations of the three-dimensional Reissner model of initially curved and twisted beam. The simplification to the initially straight, axially loaded beam studied here is made only after the consis- 
tent linearization of the governing equations has been performed. The governing equations consist of consititutive (1)-(2), equilibrium (3)-(4) and kinematic (5)-(6) equations, as presented below:

$$
\begin{aligned}
& \boldsymbol{f}_{1}=\boldsymbol{N}_{g}(x)-\mathbf{R}(x) \mathcal{C}_{N}\left(\gamma_{G}(x), \boldsymbol{\kappa}_{G}(x)\right)=\mathbf{0} \\
& \boldsymbol{f}_{2}=\boldsymbol{M}_{g}(x)-\mathbf{R}(x) \mathcal{C}_{M}\left(\gamma_{G}(x), \boldsymbol{\kappa}_{G}(x)\right)=\mathbf{0} \\
& \boldsymbol{f}_{3}=\boldsymbol{N}_{g}^{\prime}(x)+\boldsymbol{n}_{g}(x)=\mathbf{0} \\
& \boldsymbol{f}_{4}=\boldsymbol{M}_{g}^{\prime}(x)+\boldsymbol{m}_{g}(x)-\boldsymbol{N}_{g}(x) \times \boldsymbol{r}_{g}^{\prime}(x)=\mathbf{0} \\
& \boldsymbol{f}_{5}=\boldsymbol{r}_{g}^{\prime}(x)-\mathbf{R}(x)\left(\gamma_{G}(x)-\boldsymbol{c}_{G}(x)\right)=\mathbf{0} \\
& \boldsymbol{f}_{6}=\boldsymbol{\vartheta}_{g}^{\prime}(x)-\mathbf{T}^{-\mathrm{T}}(x)\left(\boldsymbol{\kappa}_{G}(x)-\boldsymbol{d}_{G}(x)\right)=\mathbf{0} .
\end{aligned}
$$

The related boundary conditions at ends $x=0$ and $x=L$ of the beam are:

$$
\begin{aligned}
& \boldsymbol{b}_{1}=\boldsymbol{F}^{0}+\boldsymbol{N}_{g}(0)=\mathbf{0} \\
& \boldsymbol{b}_{2}=\boldsymbol{P}^{0}+\boldsymbol{M}_{g}(0)=\mathbf{0} \\
& \boldsymbol{b}_{3}=\boldsymbol{F}^{L}-\boldsymbol{N}_{g}(L)=\mathbf{0} \\
& \boldsymbol{b}_{4}=\boldsymbol{P}^{L}-\boldsymbol{M}_{g}(L)=\mathbf{0} .
\end{aligned}
$$

In the above equations the following notations have been used:

$\boldsymbol{g}$ fixed (or global) orthonormal basis $\left\{\boldsymbol{g}_{1}, \boldsymbol{g}_{2}, \boldsymbol{g}_{3}\right\}$ defining the global coordinate system (Figure 1);

$\boldsymbol{G}$ local orthonormal basis $\left\{\boldsymbol{G}_{1}, \boldsymbol{G}_{2}, \boldsymbol{G}_{3}\right\}$ with $\left\{\boldsymbol{G}_{2}, \boldsymbol{G}_{3}\right\}$ spanning the rotated section;

$\boldsymbol{N}_{g}, \boldsymbol{M}_{g}$ resultant force and moment vectors of the cross-section;

$\mathcal{C}_{N}, \mathcal{C}_{M}$ operators describing material properties of a column;

$\gamma_{G}$ translational strain vector $\left(\gamma_{G 1}\right.$ is the extensional strain, $\gamma_{G 2}, \gamma_{G 3}$ are shear strains);

$\kappa_{G}$ rotational strain vector $\left(\kappa_{G 1}\right.$ is the torsional strain, $\kappa_{G 2}, \kappa_{G 3}$ are bending strains); 
$\boldsymbol{r}_{g}$ position vector of the line of centroids;

$\boldsymbol{\vartheta}_{g}$ rotational vector describing the rotation between $\left\{\boldsymbol{g}_{1}, \boldsymbol{g}_{2}, \boldsymbol{g}_{3}\right\}$ and $\left\{\boldsymbol{G}_{1}, \boldsymbol{G}_{2}, \boldsymbol{G}_{3}\right\}$;

$\vartheta$ angle of rotation, $\vartheta=\left\|\boldsymbol{\vartheta}_{g}\right\| ;$

$\mathbf{R}$ rotation matrix representing the same rotation as $\boldsymbol{\vartheta}_{g}$

$\left(\mathbf{R} \boldsymbol{u}=\boldsymbol{u}+\frac{\sin \vartheta}{\vartheta} \boldsymbol{\vartheta}_{g} \times \boldsymbol{u}+\frac{1-\cos \vartheta}{\vartheta^{2}} \boldsymbol{\vartheta}_{g} \times\left(\boldsymbol{\vartheta}_{g} \times \boldsymbol{u}\right), \boldsymbol{u}\right.$ is an arbitrary vector $) ;$

$\boldsymbol{T}^{\mathrm{T}}$ transformation matrix between $\boldsymbol{\kappa}_{G}$ and $\boldsymbol{\vartheta}_{g}^{\prime}$

$\left(\mathbf{T}^{\mathrm{T}} \mathbf{u}=\mathbf{u}-\frac{1-\cos \vartheta}{\vartheta^{2}} \boldsymbol{\vartheta}_{g} \times \boldsymbol{u}+\frac{\vartheta-\sin \vartheta}{\vartheta^{3}} \boldsymbol{\vartheta}_{g} \times\left(\boldsymbol{\vartheta}_{g} \times \boldsymbol{u}\right)\right)$

$\boldsymbol{c}_{G}, \boldsymbol{d}_{G}$ variational constants, determined from the known strains and kinematic fields of the undeformed column;

$\boldsymbol{n}_{g}, \boldsymbol{m}_{g}$ external distributed force and moment vectors per unit of the undeformed length of the axis;

$\boldsymbol{F}^{0}, \boldsymbol{F}^{L}$ external boundary point loads at $x=0, x=L$;

$\boldsymbol{P}^{0}, \boldsymbol{P}^{L}$ external boundary point moments at $x=0, x=L$.

Any vector in the above presented list can be expressed with respect to either of the two bases; the index ( $g$ or $G$ ) denotes the basis used. The rotation matrix also represents the coordinate transformation between the descriptions, i.e. $\boldsymbol{u}_{g}=\mathbf{R} \boldsymbol{u}_{G}$.

\subsection{Linearization of equations}

Eqs. (1)-(6) represent 18 scalar functions of 6 vector quantities $\boldsymbol{r}_{g}(x)$, $\boldsymbol{\vartheta}_{g}(x), \boldsymbol{N}_{g}(x), \boldsymbol{M}_{g}(x), \boldsymbol{\gamma}_{G}(x)$ and $\boldsymbol{\kappa}_{G}(x)$. If we use the notation $\boldsymbol{y}=\left[y_{1}, y_{2}, \ldots, y_{18}\right]$ for the 18-dimensional vector of all unknown functions, the linearization of the component $f_{i, j}$ function $\boldsymbol{f}_{i}$ around a fixed value $\boldsymbol{y}_{0}$ can be written as

$$
\delta f_{i, j}=\left.\sum_{k=1}^{24} \frac{\partial f}{\partial y_{k}}\right|_{\boldsymbol{y}_{0}} \delta y_{k}
$$


where $i=1, \ldots, 6, j=1,2,3 . \quad \delta y_{k}, k=1, \ldots, 18$ are arbitrary variations. They are obtained by the solution of the set of linear equations

$$
\left.\delta f_{i, j}\right|_{\boldsymbol{y}_{0}}=-f_{i, j}\left(\boldsymbol{y}_{0}\right)
$$

$(i=1, \ldots, 6, j=1,2,3)$, which is commonly expressed as

$$
\mathbf{K}\left(\boldsymbol{y}_{0}\right) \delta \boldsymbol{y}=-f\left(\boldsymbol{y}_{0}\right)
$$

$\mathbf{K}\left(\boldsymbol{y}_{0}\right)$ is the so-called tangent stiffness matrix evaluated at $\boldsymbol{y}_{0}$, or, in mathematics, the Jacobian matrix. $-f\left(\boldsymbol{y}_{0}\right)$ is the right-hand side. The above given linearization holds true only in linear vector spaces. However, the threedimensional rotations are not vectors. Therefore a special treatment is needed in the linearization of the rotation matrix $\mathbf{R}$. A further discussion regarding the linearization of $\mathbf{R}$ is beyond the scope of this article and only the result is stated:

$$
\delta \mathbf{R} u=\delta \boldsymbol{\vartheta}_{g} \times \mathbf{R} \boldsymbol{u}
$$

where $\delta \mathbf{R}$ is the variation of the rotation matrix, $\delta \boldsymbol{\vartheta}_{g}$ is the variation of the rotational vector, and $\boldsymbol{u}$ is an arbitrary vector.

For further details the reader is refered to [2] and [7].

Similarly, the linearization of the constitutive equations is represented by:

$$
\begin{aligned}
\delta \mathbf{C}_{N} & =\mathbf{C}_{\gamma \gamma} \delta \gamma_{G}+\mathbf{C}_{\gamma \kappa} \delta \boldsymbol{\kappa}_{G} \\
\delta \mathbf{C}_{M} & =\mathbf{C}_{\kappa \gamma} \delta \gamma_{G}+\mathbf{C}_{\kappa \kappa} \delta \boldsymbol{\kappa}_{G}
\end{aligned}
$$

where the components of matrices $\mathbf{C}_{\gamma \gamma}, \mathbf{C}_{\gamma \kappa}, \mathbf{C}_{\kappa \gamma}$, and $\mathbf{C}_{\kappa \kappa}$ are (see [17]) partial derivatives of $\mathbf{C}_{N}$ in $\mathbf{C}_{M}$ with respect to the components of vectors $\gamma_{G}$ in $\kappa_{G}$.

$$
\text { Matrix } C=\left[\begin{array}{cc}
\mathbf{C}_{\gamma \gamma} & \mathbf{C}_{\gamma \kappa} \\
\mathbf{C}_{\kappa \gamma} & \mathbf{C}_{\kappa \kappa}
\end{array}\right] \text { is called the cross-sectional constitutive tangent }
$$


matrix. Considering (14) gives the linearization of (3)-(6) as:

$$
\begin{aligned}
\delta \boldsymbol{f}_{3} & =\delta \boldsymbol{N}_{g}^{\prime} \\
\delta \boldsymbol{f}_{4} & =\delta \boldsymbol{M}_{g}^{\prime}-\delta \boldsymbol{N}_{g} \times \boldsymbol{r}_{g}^{\prime}-\boldsymbol{N}_{g} \times \delta \boldsymbol{r}_{g}^{\prime} \\
\delta \boldsymbol{f}_{5} & =\delta \boldsymbol{r}_{g}^{\prime}-\delta \boldsymbol{\vartheta}_{g} \times \mathbf{R}\left(\boldsymbol{\gamma}_{G}-\boldsymbol{c}_{G}\right)-\mathbf{R} \delta \boldsymbol{\gamma}_{G} \\
\delta \boldsymbol{f}_{6} & =\delta \boldsymbol{\vartheta}_{g}^{\prime}-\mathbf{R} \delta \boldsymbol{\kappa}_{G} .
\end{aligned}
$$

It has been assumed that the vectors of external loadings, $\boldsymbol{n}_{g}$ and $\boldsymbol{m}_{g}$, are independent of the unknown functions. Although the variation of equation (6) is not straightforward, the details are omitted here because they can be found in [23] or [7].

Note that Eqs. (1)-(6) consist of two algebraic equations (1)-(2) and four ordinary differential equations (3)-(6). In solving system of mixed algebraicdifferential equations, it is suitable to eliminate the algebraic part. This is formally done by expressing the strains from the inverse of the constitutive equations (1)-(2), yet such an approach is not always unique, e. g. when the inverse does not exist.

Eqs. (1)-(2) will therefore be varied first and then $\delta \gamma_{G}$ and $\delta \boldsymbol{\kappa}_{G}$ expressed by inverting the linearized equations. Considering (14)-(16) yields

$$
\begin{aligned}
& \delta \boldsymbol{f}_{1}=\delta \boldsymbol{\vartheta}_{g} \times \mathbf{R C}_{N}+\mathbf{R} \mathbf{C}_{\gamma \gamma} \delta \gamma_{G}+\mathbf{R C}_{\gamma \kappa} \delta \boldsymbol{\kappa}_{G}-\delta \boldsymbol{N}_{g} \\
& \delta \boldsymbol{f}_{2}=\delta \boldsymbol{\vartheta}_{g} \times \mathbf{R} \mathbf{C}_{M}+\mathbf{R} \mathbf{C}_{\kappa \gamma} \delta \boldsymbol{\gamma}_{G}+\mathbf{R} \mathbf{C}_{\kappa \kappa} \delta \boldsymbol{\kappa}_{G}-\delta \boldsymbol{M}_{g}
\end{aligned}
$$

From (12) it follows

$$
\begin{aligned}
& \delta \boldsymbol{f}_{1}=\boldsymbol{N}_{g}-\mathbf{R C}_{N} \\
& \delta \boldsymbol{f}_{2}=\boldsymbol{M}_{g}-\mathbf{R C}_{M} .
\end{aligned}
$$

It is further assumed that, in an arbitrary configuration, the constitutive equations are fulfilled, i.e. $\boldsymbol{N}_{g}=\mathbf{R} \mathbf{C}_{N}$ and $\boldsymbol{M}_{g}=\mathbf{R} \mathbf{C}_{M}$. From (21)-(24) we can then express:

$$
\begin{gathered}
\delta \boldsymbol{\gamma}_{G}=\mathbf{C}_{\gamma \gamma}^{-1} \mathbf{R}^{\mathrm{T}}\left(\delta \boldsymbol{N}_{g}-\delta \boldsymbol{\vartheta}_{g} \times \boldsymbol{N}_{g}\right)+\mathbf{C}_{\kappa \gamma}^{-1} \mathbf{R}^{\mathrm{T}}\left(\delta \boldsymbol{M}_{g}-\delta \boldsymbol{\vartheta}_{g} \times \boldsymbol{M}_{g}\right) \\
\delta \boldsymbol{\kappa}_{G}=\mathbf{C}_{\kappa \gamma}^{-1} \mathbf{R}^{\mathrm{T}}\left(\delta \boldsymbol{N}_{g}-\delta \boldsymbol{\vartheta}_{g} \times \boldsymbol{N}_{g}\right)+\mathbf{C}_{\kappa \kappa}^{-1} \mathbf{R}^{\mathrm{T}}\left(\delta \boldsymbol{M}_{g}-\delta \boldsymbol{\vartheta}_{g} \times \boldsymbol{M}_{g}\right) .
\end{gathered}
$$


The linearization of the boundary conditions (7)-(10) is straightforward and reads:

$$
\begin{aligned}
\delta \boldsymbol{b}_{1} & =-\delta \boldsymbol{N}_{g}(0) \\
\delta \boldsymbol{b}_{2} & =-\delta \boldsymbol{M}_{g}(0) \\
\delta \boldsymbol{b}_{3} & =-\delta \boldsymbol{N}_{g}(L) \\
\delta \boldsymbol{b}_{4} & =-\delta \boldsymbol{M}_{g}(L) .
\end{aligned}
$$

\subsection{Analytical solution of the linearized equations}

The linearized equations are evaluated at the fundamental configuration, which is in our case an initially straight axially-loaded column with an existing, yet still closed delamination, as presented in Section 2. It is assumed that the local and the global coordinate systems coincide initially. At the initial state, all the quantities describing the beam are known. From the above assumptions it follows that, in the fundamental configuration,

$$
\begin{array}{lc}
\boldsymbol{\vartheta}_{g, 0}(x)=\mathbf{0}, & \mathbf{R}_{0}(x)=\mathbf{I}, \\
\boldsymbol{n}_{g}(x)=\mathbf{0}, \quad \boldsymbol{m}_{g}(x)=\mathbf{0}, \\
\boldsymbol{P}^{0}=[0,0,0]^{\mathrm{T}}, \quad \boldsymbol{P}^{L}=[0,0,0]^{\mathrm{T}}, \\
\boldsymbol{c}_{G}=[1,0,0]^{\mathrm{T}}, \quad \boldsymbol{d}_{G}=[0,0,0]^{\mathrm{T}}, \\
\boldsymbol{r}_{0}(x)=[x, 0,0]^{\mathrm{T}} \\
\boldsymbol{F}^{0}=[F, 0,0]^{\mathrm{T}} & \boldsymbol{F}^{L}=[-F, 0,0]^{\mathrm{T}} .
\end{array}
$$

In such conditions, the curvatures $\boldsymbol{\kappa}_{0}(x)$ and internal moments $\boldsymbol{M}_{g}(x)$ are also zero in the fundamental configuration. Consequently, the translational strains and the boundary forces are constant taking the forms $\gamma_{g}=\gamma_{G}=$ $\left[\begin{array}{lll}\gamma_{1}^{i} & 0 & 0\end{array}\right]^{\mathrm{T}}$ and $\boldsymbol{N}_{g}=\left[\begin{array}{lll}N_{1}^{i} & 0 & 0\end{array}\right]^{\mathrm{T}}$, where $i=\{a, b, c, d\}$. As the cross-section is

assumed constant, $\gamma_{1}^{a}=\gamma_{1}^{b}=\gamma_{1}^{c}=\gamma_{1}^{d}$ and $A^{b}+A^{c}=A^{a}=A^{d}$. The axial forces 
of the elements are then given by

$$
\begin{aligned}
& N_{1, g}^{a}=N_{1, g}^{d}=-F \\
& N_{1, g}^{b}=-\frac{A^{b}}{A^{d}} F \\
& N_{1, g}^{c}=-\frac{A^{c}}{A^{d}} F .
\end{aligned}
$$

After inserting (25)-(26) and (31)-(39) in (3)-(10), (17)-(20) and (27)-(30), the linearized system (12) of the present problem reads

$$
\begin{aligned}
& \delta \boldsymbol{N}_{g}^{\prime}=0 \\
& \delta \boldsymbol{M}_{g}^{\prime}-\delta \boldsymbol{N}_{g} \times \boldsymbol{r}_{g}^{\prime}-\boldsymbol{N}_{g} \times \delta \boldsymbol{r}_{g}^{\prime}=\boldsymbol{M}_{g, 0}^{\prime}-\boldsymbol{N}_{g, 0} \times \boldsymbol{r}_{g, 0}^{\prime} \\
& \delta \boldsymbol{r}_{g}^{\prime}-\delta \boldsymbol{\vartheta}_{g} \times \boldsymbol{r}_{g}^{\prime}-\mathbf{R}\left(\mathbf{C}_{\gamma \gamma}^{-1} \mathbf{R}^{\mathrm{T}}\left(\delta \boldsymbol{N}_{g}-\delta \boldsymbol{\vartheta}_{g} \times \boldsymbol{N}_{g}\right)\right. \\
& \left.-\mathbf{C}_{\kappa \gamma}^{-1} \mathbf{R}^{\mathrm{T}}\left(\delta \boldsymbol{M}_{g}-\delta \boldsymbol{\vartheta}_{g} \times \boldsymbol{M}_{g}\right)\right)=\boldsymbol{r}_{g, 0}^{\prime}-\mathbf{R}\left(\boldsymbol{\gamma}_{G, 0}-\boldsymbol{c}_{G}\right) \\
& \delta \boldsymbol{\vartheta}_{g}^{\prime}-\mathbf{R}\left(\mathbf{C}_{\kappa \gamma}^{-1} \mathbf{R}^{\mathrm{T}}\left(\delta \boldsymbol{N}_{g}-\delta \boldsymbol{\vartheta}_{g} \times \boldsymbol{N}_{g}\right)\right. \\
& \left.-\mathbf{C}_{\kappa \kappa}^{-1} \mathbf{R}^{\mathrm{T}}\left(\delta \boldsymbol{M}_{g}-\delta \boldsymbol{\vartheta}_{g} \times \boldsymbol{M}_{g}\right)\right)=\boldsymbol{\vartheta}_{g, 0}^{\prime}-\mathbf{T}^{-\mathrm{T}}\left(\boldsymbol{\kappa}_{G, 0}-\boldsymbol{d}_{G}\right) \\
& \delta \boldsymbol{N}_{g}(0)=[-F, 0,0]^{\mathrm{T}}-\boldsymbol{N}_{g}(0) \\
& \delta \boldsymbol{M}_{g}(0)=0 \\
& -\delta \boldsymbol{N}_{g}(L)=-[-F, 0,0]^{\mathrm{T}}+\boldsymbol{N}_{g}(L) \\
& \delta \boldsymbol{M}_{g}(L)=0 .
\end{aligned}
$$

The fundamental configuration should be in keeping with the kinematic equations (5)-(6). Therefore the right-hand sides in Eqs. (42)-(43) automatically vanish. The load in the fundamental configuration points in the axial direction, thus vectors $\boldsymbol{N}_{g, 0}$ and $\boldsymbol{r}_{g, 0}$ remain parallel and their vector product equals zero.

The system of equations (40)-(43) is thus a homogeneous system of linear differential equations of the first order with constant coefficients. It can be expressed in the matrix form

$$
\delta \boldsymbol{y}^{\prime}(x)=\boldsymbol{B} \delta \boldsymbol{y}(x)
$$


where $\boldsymbol{B}$ represents the system matrix of constant coefficients and $\delta \boldsymbol{y}$ is the vector of unknowns. The related static boundary conditions (44)-(47) and kinematic boundary conditions are discussed in the next subsection.

The analytical solution of the non-homogeneous system of differential equations (48) for a constant matrix $\boldsymbol{B}$ can be found in, e. g. [9]:

$$
\delta \boldsymbol{y}(x)=e^{\boldsymbol{B} x} \boldsymbol{\beta},
$$

where $\boldsymbol{\beta}$ is a 12-dimensional vector of the integration constants that must be determined from the given boundary conditions of the column.

The solution given in Eq. (49) holds for each element. It can be interpreted as having exact shape functions for 12 unknown scalar constants of $\boldsymbol{\beta}$. Our mathematical model of the delaminated beam thus consists of four elements with known analytical solutions requiring 12 unknown scalar constants each. They are derived from the boundary conditions as discribed next.

\subsection{Boundary conditions}

Various discrete support types at the boundaries of the column will be considered. We also need to prescribe the conditions between the delaminated part of the column and the non-delaminated ends. These internal conditions are often called the continuity conditions; in fact, they require the continuity of displacements and rotations, and that the equilibrium of internal forces is satisfied.

At the two points of the neutral axis of the column (points $T_{1}$ and $T_{2}$ in Figure 1) where the external layers bond to the undelaminated ends we have

$$
\begin{gathered}
\delta \boldsymbol{r}_{g}^{a}\left(L_{1}\right)=\delta \boldsymbol{r}_{g}^{b}(0)=\delta \boldsymbol{r}_{g}^{c}(0) \\
\delta \boldsymbol{r}_{g}^{b}\left(L_{23}\right)=\delta \boldsymbol{r}_{g}^{c}\left(L_{23}\right)=\delta \boldsymbol{r}_{g}^{d}(0) \\
\delta \boldsymbol{\vartheta}_{g}^{a}\left(L_{1}\right)=\delta \boldsymbol{\vartheta}_{g}^{b}(0)=\delta \boldsymbol{\vartheta}_{g}^{c}(0) \\
\delta \boldsymbol{\vartheta}_{g}^{b}\left(L_{23}\right)=\delta \boldsymbol{\vartheta}_{g}^{c}\left(L_{23}\right)=\delta \boldsymbol{\vartheta}_{g}^{d}(0)
\end{gathered}
$$


and

$$
\begin{gathered}
\delta \boldsymbol{N}_{g}^{a}\left(L_{1}\right)=\delta \boldsymbol{N}_{g}^{b}(0)+\delta \boldsymbol{N}_{g}^{c}(0) \\
\delta \boldsymbol{N}_{g}^{b}\left(L_{23}\right)+\delta \boldsymbol{N}_{g}^{c}\left(L_{23}\right)=\delta \boldsymbol{N}_{g}^{d}(0) \\
\delta \boldsymbol{M}_{g}^{a}\left(L_{1}\right)=\delta \boldsymbol{M}_{g}^{b}(0)+\delta \boldsymbol{M}_{g}^{c}(0) \\
\delta \boldsymbol{M}_{g}^{b}\left(L_{23}\right)+\delta \boldsymbol{M}_{g}^{c}\left(L_{23}\right)=\delta \boldsymbol{M}_{g}^{d}(0) .
\end{gathered}
$$

Eqs. (50)-(57) represent 36 scalar conditions. The remaining 12, yet unspecified conditions follow from the conditions provided by the supports.

Simply-supported beam. In the three-dimensional model, only the rotation about the lateral axis is allowed. The displacement is allowed only in the axial direction at one support. Static boundary conditions follow directly from (44)(47). The complete set of independent boundary conditions for this case is:

$$
\begin{array}{r}
\delta \vartheta_{1}^{a}(0)=\delta \vartheta_{2}^{a}(0)=0 \\
\delta M_{3}^{a}(0)=0 \\
\delta \vartheta_{1}^{d}\left(L_{4}\right)=\delta \vartheta_{2}^{d}\left(L_{4}\right)=0 \\
\delta M_{3}^{d}\left(L_{4}\right)=0 \\
\delta r_{a}^{1}(0)=\delta r_{a}^{2}(0)=\delta r_{a}^{3}(0)=0 \\
\delta r_{d}^{2}\left(L_{4}\right)=\delta r_{d}^{3}\left(L_{4}\right)=0 \\
\delta N_{d}^{1}\left(L_{4}\right)=0 .
\end{array}
$$

Cantilever beam. Here the column is clamped at one end and loaded with the axial force at the other. The boundary conditions are:

$$
\begin{array}{r}
\delta r_{a}^{1}(0)=\delta r_{a}^{2}(0)=\delta r_{a}^{3}(0)=0 \\
\delta \vartheta_{1}^{a}(0)=\delta \vartheta_{2}^{a}(0)=\delta \vartheta_{3}^{a}(0)=0 \\
\delta N_{d}^{1}\left(L_{4}\right)=\delta N_{d}^{2}\left(L_{4}\right)=\delta N_{d}^{3}\left(L_{4}\right)=0 \\
\delta M_{1}^{d}\left(L_{4}\right)=\delta M_{2}^{d}\left(L_{4}\right)=\delta M_{3}^{d}\left(L_{4}\right)=0 .
\end{array}
$$

The total set of scalar conditions can be written as a homogeneous system of 
algebraic equations

$$
\boldsymbol{K} \boldsymbol{\beta}=\mathbf{0},
$$

where $\boldsymbol{K}$ stands for the matrix of coefficients, and $\boldsymbol{\beta}$ is the vector of constants. We are interested in non-trivial solutions for $\delta \boldsymbol{y}$, thus $\boldsymbol{\beta}$ must be non-zero. In order to obtain the non-trivial solutions, matrix $\boldsymbol{K}$ must be singular.

As the only parameter left is the magnitude of the axial force $F$, the lowest value for $F$ is sought such that the matrix $\boldsymbol{K}$ becomes singular. For further details on the calculation of the critical points and their classification see Planinc and Saje [14].

\section{Results and discussion}

In order to make comparisons with the results from literature possible, we will limit the present numerical studies to the linear elastic material. Then the operators $\boldsymbol{C}_{N}$ and $\boldsymbol{C}_{M}$ take the forms:

$$
\begin{gathered}
\boldsymbol{C}_{N}\left(\boldsymbol{\gamma}_{G}, \boldsymbol{\kappa}_{G}\right)=\left[\begin{array}{cccccc}
E A & 0 & 0 & 0 & E S_{2} & -E S_{3} \\
0 & G A_{2} & 0 & 0 & 0 & 0 \\
0 & 0 & G A_{3} & 0 & 0 & 0
\end{array}\right] \gamma_{G} \\
\boldsymbol{C}_{M}\left(\gamma_{G}, \boldsymbol{\kappa}_{G}\right)=\left[\begin{array}{cccccc}
0 & 0 & 0 & G I_{t} & 0 & 0 \\
E S_{2} & G A_{2} & 0 & 0 & E I_{2} & E I_{23} \\
-E S_{3} & 0 & G A_{3} & 0 & E I_{23} & E I_{3}
\end{array}\right] \boldsymbol{\kappa}_{G} .
\end{gathered}
$$

$E$ and $G$ denote elastic and shear moduli of material; $A$ is the cross-sectional area; $S_{2}$ and $S_{3}$ are the two moments of area with respect to local axes; $I_{t}$ is the torsional inertial moment of the cross-section; $A_{2}$ and $A_{3}$ are the effective shear areas in the directions of $\boldsymbol{G}_{2}$ and $\boldsymbol{G}_{3} ; I_{2}$ and $I_{3}$ are the inertial moments, and $I_{23}$ is the deviatoric inertial moment of the cross-section with respect to local coordinates on the reference axis.

Results are presented for different values of ratio between elastic and shear moduli greater or equal to 2 , which corresponds to materials like concrete, steel or timber, and anisotropic polymer materials. 
We analyze the effects of the delamination length, delamination position, shear modulus and slenderness ratio on the buckling load.

The critical force $\left(F_{\text {cr }}\right)$ is normalized with respect to the classical Euler's buckling force $\left(F_{\mathrm{E}}\right)$ of each considered case. Most of the results are presented using the slenderness of the column defined as:

$$
\lambda=L \sqrt{\frac{A}{I}} .
$$

Here $I$ denotes the smallest value of the cross-sectional inertial moments.
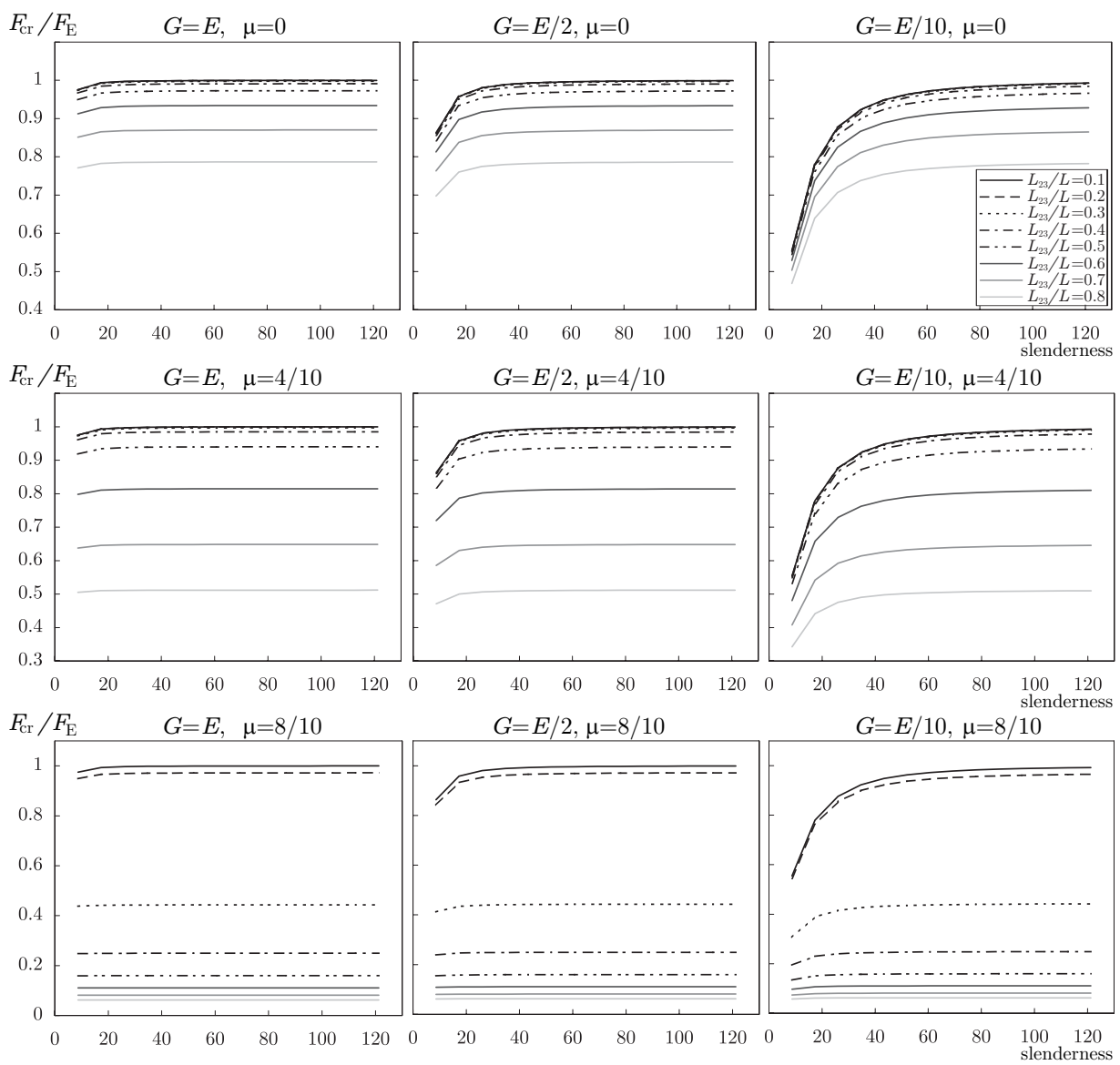

Figure 3: Simply supported column: relative critical force vs. slenderness for various delamination lengths $\left(L_{23} / L\right)$, shear moduli $(G)$ and delamination positions $(\mu)$. 
We consider a straight simply-supported delaminated column with a constant cross-section, having height $h=20 \mathrm{~cm}$ and width $b=40 \mathrm{~cm}$. Our first analysis assumes the variation of the length of delamination $L_{23}$ with respect to the total length $L$ of the column and considers three different material moduli ratios $G / E=1,1 / 2,1 / 10$. For each chosen combination, the results are shown as a function of the slenderness of the column, varying it from thick to slender.

Figure 3 presents the relative critical force vs. slenderness for various relative delamination lengths $\left(L_{23} / L\right)$, elastic to shear moduli ratios $(E / G)$ and position of the delamination with respect to the cross-section $(\mu)$.

The delaminations in Fig. 3 are taken to be parallel with edges of the cross-section $(\phi=0)$. The large effect of elastic-to-shear-moduli ratios $E / G$ on the magnitude of the critical force is evident from Figure 3. Small values of $G / E$ result in substantially smaller critical forces for low values of slenderness. As expected, the buckling force is also highly dependent on the delamination length. Longer delaminations significantly lower the buckling force. Note also a major influence of an asymmetrical position of the delamination with respect to the height of the cross-section. The magnitude of the buckling force reduces significantly when the delamination is close to the edge of the column.

Fig. 4 presents relative critical force (for several discrete values of slenderness $\lambda)$ vs. the relative delamination length $\left(L_{23} / L\right)$ and the longitudinal position
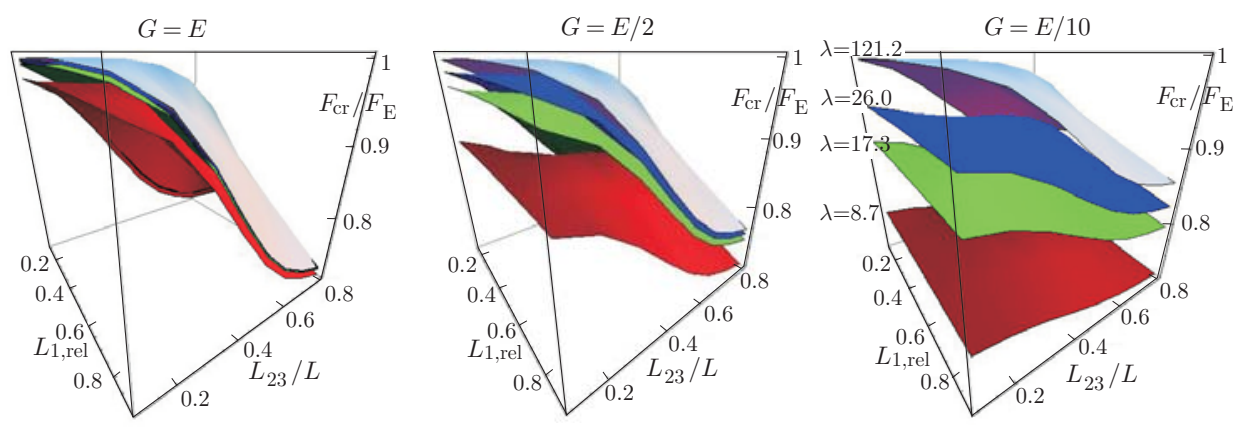

Figure 4: Simply supported column: relative critical force vs. relative delamination length $\left(L_{23} / L\right)$ and relative longitudinal position of delamination $\left(L_{1, \mathrm{rel}}\right)$. 
of the delamination, defined as the relative length of the first non-delaminated element $L_{1, \text { rel }}=L_{1} /\left(L-L_{23}\right)$.

Values of $L_{1, \text { rel }}$ around 0.5 (describing the central delamination) increase the magnitudes of the critical force. The increase of the length of the delamination makes the critical forces smaller.

Fig. 5 depicts the relative critical force for several discrete values of the slendernesses of the column vs. the relative delamination length and asymmetrical position $\mu$ of delamination according to the height of cross-section. It can be observed that the increased lateral asymmetry significantly reduces the critical force. This is particularly pronounced in the case of large delamination lengths. The reduction of the critical force as a result of a low ratio $G / E$ can also be observed.
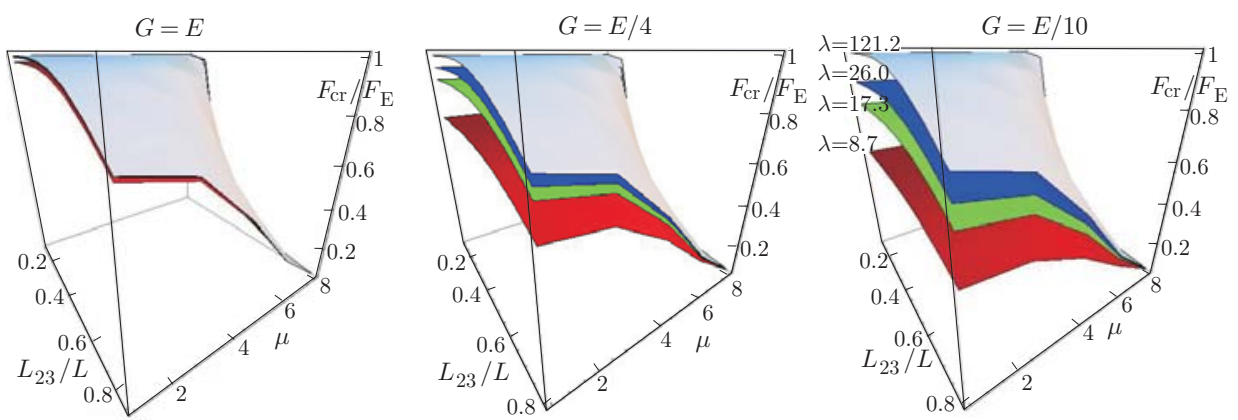

Figure 5: Relative critical force vs. relative delamination length and relative lateral asymmetrical position $\mu$ of delamination.

Our last example presents a cantilever beam with a constant cross-section, having height $h=40 \mathrm{~cm}$ and width $b=20 \mathrm{~cm}$. The length of the column is $L=400 \mathrm{~cm}$. The objective of this analysis is to show how the magnitude of the critical force $\left(F_{\mathrm{cr}}\right)$ depends on the rotation of delamination $\phi$ for various asymmetrical positions of delamination $\mu$ and for different lengths of delamination $L_{23}$, as it is most significant for this boundary conditions. Results are presented in Figure 6 in the form of polar graphs showing the relative critical force vs. the 
angle of delamination.
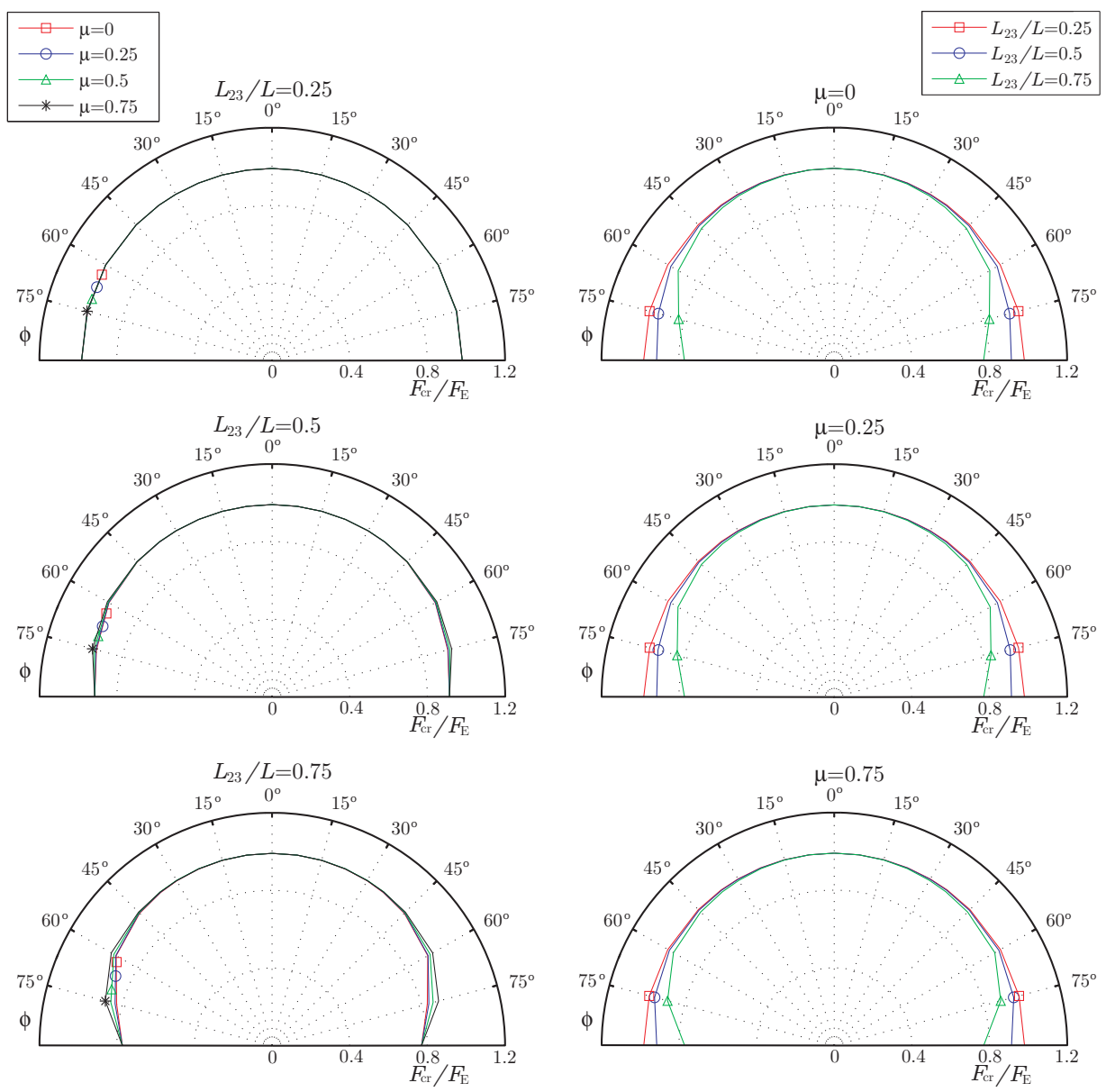

Figure 6: Normalized relative critical force $\left(F_{\mathrm{cr}} / F_{\mathrm{E}}\right)$ vs. rotation of delamination $\phi$ (for different values of asymmetrical position of delamination $\mu$ and for different lengths of delamination $\left.L_{23}\right)$.

By increasing the angle of delamination $\phi$, we can observe some reduction of the relative critical forces. This effect becomes considerable only for large angles (more than $30^{\circ}$ ) and longer delaminations. For relatively short delaminations $\left(L_{23} / L=0.25\right)$, the effects of the lateral asymmetry and the angle of delamination can safely be neglected. While in the present analysis the slenderness 
of the column was taken to be 34.6 , the increase of the slenderness changes the results only slightly.

Last two figures present buckling modes for typical angles $\phi$ of a cantilever beam with the position of delamination $\mu=0$ (Fig.7) and $\mu=0.75$ (Fig.8).

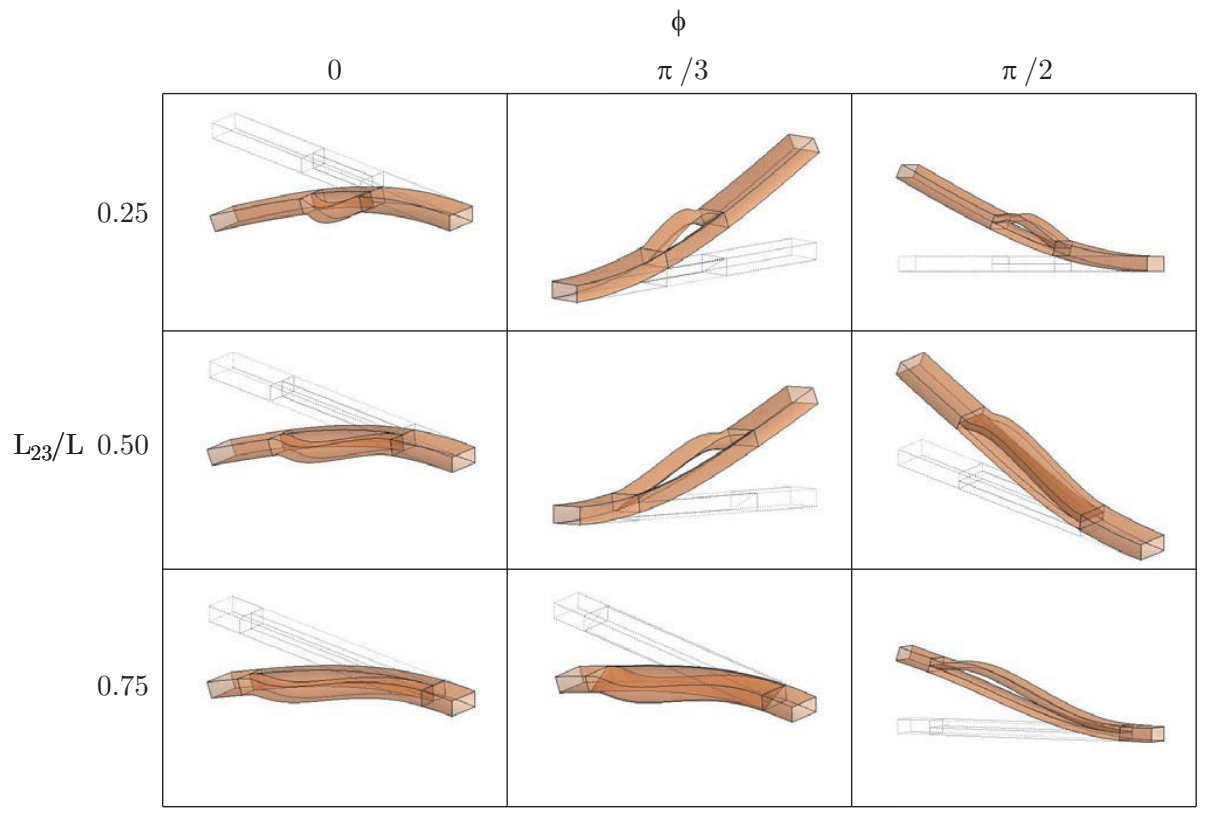

Figure 7: Buckling modes for typical angles $\phi$ of a cantilever beam with the position of delamination $\mu=0$ and varying length of delamination $L_{23} / L$.

We compare the results of the present forumulation with already known results from literature. In the first example we compare results for the relative vertical position $r_{d}=0.4$ and various lengths of the delamination $\left(l_{d}=\right.$ $0.2,0.4,0.6,0.8)$ with results obtained by the Abaqus finite element code [12], Simitses et al. [19] and the energy method presented by Lim and Parsons [12]. Each result is normalized with Euler's [5] buckling load for the undelaminated beam. Table 1 presents results of the normalized buckling load for the simply supported beam with slenderness ratio $\lambda=45$. The beam was delaminated with a longitudinally symmetric delamination. Young's modulus $E=3000 \mathrm{kN} / \mathrm{cm}^{2}$ 


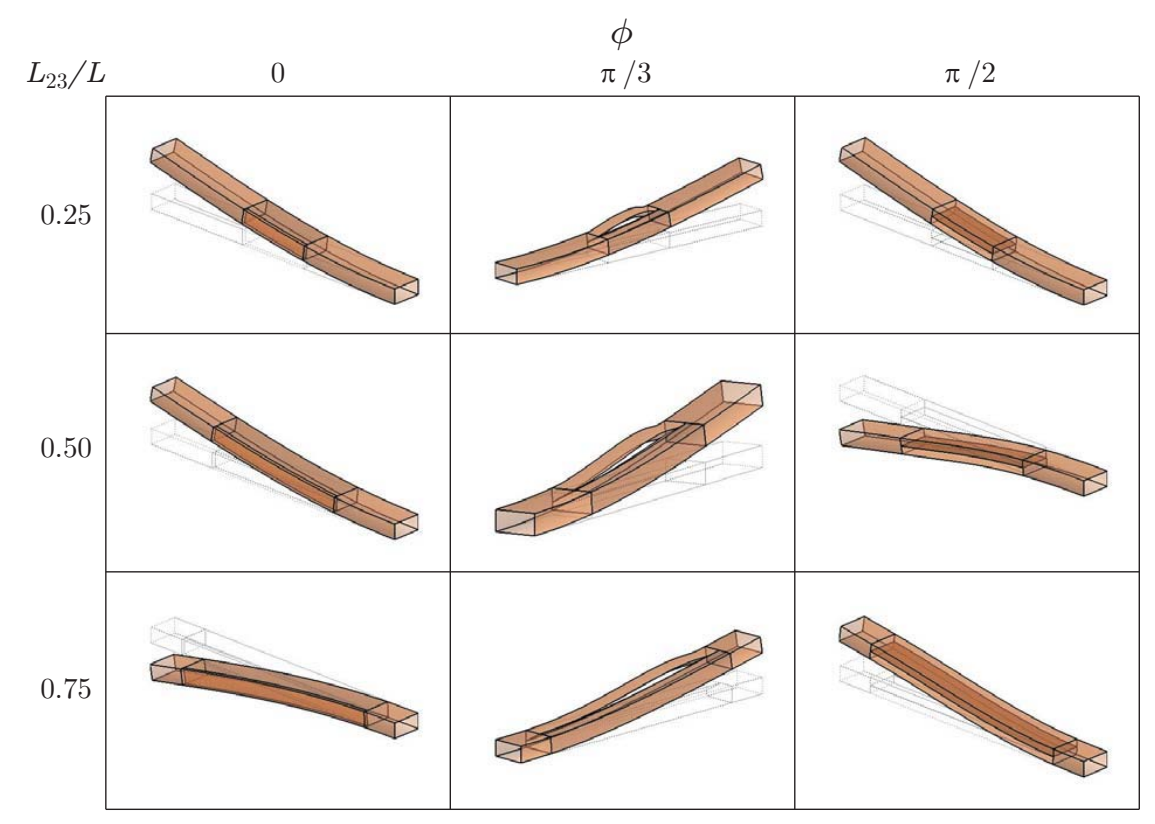

Figure 8: Buckling modes for typical angles $\phi$ of a cantilever beam with the position of delamination $\mu=0.75$ and varying length of delamination $L_{23} / L$.

was assumed.

In the second example we compare results for the clamped-clamped beam with a delamination at two different relative vertical positions $r_{d}=0.2,0.3$ and various lengths, $l_{d}$. We used the same material and geometric characteristics as in the previous case. Additionally we considered $v=0.3$ and $G=\frac{E}{2(1-v)}$. Table 2 presents results of the normalized buckling load where each result is normalized with buckling load according to Chen [4]. We compare the present results with the results of Chen [4] ('CLT'-classical lamination theory and 'SDT'-shear deformation theory) and Kardomateas and Schmueser [8].

\section{Conclusions}

An analytical solution for buckling of asymmetrically delaminated spatial columns considering shear effects is presented. The essential features of the present approach are: 
Table 1: Normalized buckling loads for simply supported beam with a single delamination relative vertical positions $r_{d}=0.4$ and various lengths, $l_{d}$.

\begin{tabular}{cccccc}
\hline Method $/ l_{d}$ & 0 & 0.2 & 0.4 & 0.6 & 0.8 \\
\hline Euler [5] & 1.0000 & - & - & - & - \\
Timoshenko [20] & 0.9715 & - & - & - & - \\
Reissner [16] & 0.9715 & - & - & - & - \\
En. method [12] & - & 0.9997 & 0.9902 & 0.9198 & 0.7264 \\
Abaqus [12] & - & 0.9997 & 0.9902 & 0.9197 & 0.7264 \\
Simitses et al. [19] & - & 0.9997 & 0.9902 & 0.9198 & 0.7264 \\
Present $(G=E / 6)$ & 0.9715 & 0.9712 & 0.9622 & 0.8956 & 0.7111 \\
Present $(G=\infty)$ & 1.0049 & 1.0046 & 0.9950 & 0.9240 & 0.7290 \\
\hline
\end{tabular}

Table 2: Normalized buckling loads of clamped-clamed beam with a single delamination at two different relative vertical positions $r_{d}=0.2,0.3$ and various lengths, $l_{d}$.

\begin{tabular}{ccccccc}
\hline$l_{d}$ & $r_{d}$ & SDT[4] & CLT[4] & Kar. and Schmu. [8] & Present $\left(\varepsilon_{\text {init. }}=0\right)$ & Present \\
\hline 0.2 & 0.2 & 0.7816 & 0.9264 & 0.8003 & 0.8003 & 0.8130 \\
& 0.3 & 0.8280 & 0.9924 & 0.8543 & 0.8543 & 0.8688 \\
\hline 0.4 & 0.2 & 0.2354 & 0.2471 & 0.2215 & 0.2215 & 0.2226 \\
& 0.3 & 0.4803 & 0.5314 & 0.4689 & 0.4689 & 0.4734 \\
\hline \multirow{2}{*}{0.6} & 0.2 & 0.1080 & 0.1103 & 0.0997 & 0.0997 & 0.1000 \\
& 0.3 & 0.2322 & 0.2435 & 0.2184 & 0.2184 & 0.2194 \\
\hline 0.8 & 0.2 & 0.0615 & 0.0623 & 0.0565 & 0.0565 & 0.0565 \\
& 0.3 & 0.1353 & 0.1390 & 0.1254 & 0.1254 & 0.1258 \\
\hline
\end{tabular}

- We are able to obtain analytically the buckling forces of a delaminated column with three-dimensional behaviour and with the transverse-shear effect being consistently taken into account.

- The present approach allows us to study arbitrary cross-sections and planar delaminations inclined with respect to the cross-sections. 
- The three-dimensional behaviour makes us possible to consider properly the cases when the global buckling of the column is not in the same direction as the local buckling of the layers.

- The transverse-shear effect is taken in line with the Reissner beam theory which considers properly the coupling of the extensional and bending stiffnesses.

- The present exact results can well serve as benchmarks for numerical models.

Some further parametric studies have also been presented which reveal that

- The dependence of the buckling load on the delamination length and the position of the delamination is non-linear.

- The effect of transverse shear can influence significally the buckling force.

- Not only the length but also the position of the delamination has a considerable effect. We have shown that the angle of the delamination plane is less important, yet not to be neglected.

\section{References}

[1] E. A. Almond, Delamination in banded steels, Metallurgical transactions 1, 2038-2041, 1970.

[2] J. H. Argyris, An excursion into large rotations, Comput. Methods Appl. Mech. Eng. 32, 85-155, 1982.

[3] H. Chai, C. D. Babcock, W. G. Knauss, One dimensional modelling of failure in laminated plates by delamination buckling, Int. J. Solids Struct. 17, 1069-1083, 1981.

[4] H. P. Chen, Shear deformation theory for compressive delamination buckling and growth, AIAA Journal 29(5), 813-819, 1991. 
[5] L. Euler, Methodus inveniendi lineas curvas maximi minimive proprietate gaudentes, Lausanne, 1744.

[6] H. Gu, A. Chattopadhyay, An experimental investigation of delamination buckling and postbuckling of composite laminates, Compos. Sci. Technol. 59, 903-910, 1999.

[7] A. Ibrahimbegovic, On the choice of finite rotation parameters, Comput. Methods Appl. Mech. Eng. 149, 49-71, 1997.

[8] G. A. Kardomateas, D. W. Schmueser, Buckling and postbuckling of delaminated composites under compressive loads including transverse shear effect, AIAA Journal 26(3), 337-343, 1988.

[9] J. L. Goldberg, A. J. Schwartz, System of ordinary differential equations: An Introduction, Harper \& Row, Publishes, New York, 1972.

[10] S.-F. Hwang, G.-H. Liu, Buckling behavior of composite laminates with multiple delaminations under uniaxial compression, Composite Structures $53,235-243,2001$.

[11] A. Kryžanovski, M. Saje, I. Planinc, D. Zupan, Analytical solution for buckling of asymmetrically delaminated Reissner's elastic columns including transverse shear, Int. J. Solids Struct. 45 (3-4), 1051-1070, 2008.

[12] Y. B. Lim, I. D. Parsons, The linearized buckling analysis of a composite beam with multiple delaminations, Int. J. Solids Struct. 30, 3085-3099, 1993.

[13] S. Moradi, F. Taheri, Delamination buckling analysis of general laminated composite beams by differential quadrature method, Composites: Part B30, 503-511, 1999.

[14] I. Planinc, M. Saje, A quadratically convergent algorithm for the computation of stability points: The application of the determinant of the tangent stiffness matrix, Comp. Meth. Appl. Mech. and Eng. 169, 89-105, 1999. 
[15] E. Reissner, On finite deformation of space-curved beams, J. Appl. Math. Phys. 32, 734-744, 1981.

[16] E. Reissner, Some remarks on the problem of Euler buckling, Ing. Archiv. $52,115-119,1982$.

[17] U. Rodman, M. Saje, I. Planinc, D. Zupan, Exact buckling analysis of composite columns including multiple delamination and transverse shear, Eng. Struct. 30, 1500-1514, 2007.

[18] G. J. Short, F. J. Guild, M. J. Pavier, The effect of delamination geometry on the compressive failure of composite laminates, Compos. Sci. Technol. 61, 2075-2086, 2001.

[19] G. J. Simitses, S. Sallam, W. L. Yin, Effect of delamination of axially loaded homogeneous laminated plates, AIAA Journal 23(9), 1437-1445, 1985.

[20] S. P. Timoshenko, J. M. Gere, Theory of elastic stability, McGraw-Hill, 1961.

[21] C. W. Yap, G. B. Chai, Analytical and numerical studies on the buckling of delaminated composite beams, Composite Structures 80, 307-319, 2007.

[22] W. L. Yin, S. Sallam, G. J. Simitses, Ultimate axial load capacity of a delaminated beam-plate, AIAA Journal 24, 123-128, 1986.

[23] D. Zupan, M. Saje, Finite-element formulation of geometrically exact threedimensional beam theories based on interpolation of strain measures, Comput. Methods Appl. Mech. Eng. 192, 5209-5248, 2003. 